\title{
SYNTHESIS AND SPECTROSCOPIC CHARACTERIZATION OF HYBRID MAGNETIC NANOPARTICLES, BASED ON FE@AU AND PYRROLE
}

\author{
MARIA-LOREDANA SORAN ${ }^{a^{*}}$, OVIDIU PANĂ ${ }^{a}$, ALEXANDRINA NAN ${ }^{a}$, \\ CRISTIAN LEOŞTEAN ${ }^{a}$, IOAN BRATUa
}

\begin{abstract}
The synthesis and characterization of Fe@Au and Fe@Au covered with pyrrole and pyrrole functionalized with $\mathrm{N}$-succinimide is presented. The synthesis of Fe@Au nanoparticles, with diameters between 5-7 nm was performed by the reverse micelles method. The hybrid composites Fe@Au nanoparticles covered by the pyrrole copolymer were obtained by chemical oxidative polymerization of a mixture of pyrrole and substituted pyrrole with $\mathrm{N}$-succinimide monomers in water. The properties of these composites were investigated by transmission electron microscopy and FTIR spectroscopy.
\end{abstract}

Keywords: Nanoparticles; Iron; Gold; Polypyrrole; N-succinimide

\section{INTRODUCTION}

Various types of magnetic nanoparticle systems have been subject to a considerable interest in the last few years from both fundamental and applicative point of view [1-3]. Generally, when the sizes of the magnetic particles are decreased to the nanometric scale, a transition occurs from polydomain magnetism to monodomain magnetic systems [4-5]. A system of noninteracting nanoparticles displays the superparamagnetism phenomenon [6].

\footnotetext{
a National Institute for Research \& Development of Isotopic and Molecular Technologies, 65-103 Donath Street, Cluj-Napoca, Romania

* Corresponding author: loredana.soran@itim-cj.ro
} 
Besides the adjustment of dimensions, additional control and design of magnetic nanoparticles could be achieved by developing core-shell structured nanosystems. This type of particles has drawn the attention since they allow the tailoring of the combined surface and core properties providing also an increased number of applications [7-9]. Among the core shell nanoparticles those formed with magnetic core and having the outer shell formed by noble metals have drown interest since one obtains an enhancement of the thermal and chemical stability of nanoparticles, an improvement of the solubility [1014]. For instance, an outer Au shell makes them less cytotoxic and allows the functionalization by attaching other molecules to the surface of the particles. In certain cases, the shell also prevents the oxidation of the core material [7]. The coating of the magnetic nanoparticles with polymers represents a new route for tailoring the properties. It could be achieved by controlling the polymer composition and structure [15]. In the present study we report the synthesis, characterization (mainly spectroscopic) and possible applications of the ironcore and gold - shell (Fe@Au) nanoparticles covered with copolymer between pyrrole and pyrrole functionalized with $\mathrm{N}$-succinimide (PPy-NHS).

\section{RESULTS AND DISCUSSION}

As an example, the TEM image of an ensemble of Fe@Au core-shell nanoparticles is shown in Fig. 1a. It corresponds to the FA1 sample from Table 1. In the Fig. $1 \mathrm{~b}$ it is presented the TEM image of the hybrid composite formed by $\mathrm{Fe} @$ Au nanoparticles and the Py/Py-NHS copolymer. One can see that also the copolymer surrounds the Fe@Au nanoparticles in a core-shell like arrangement forming globular structures.
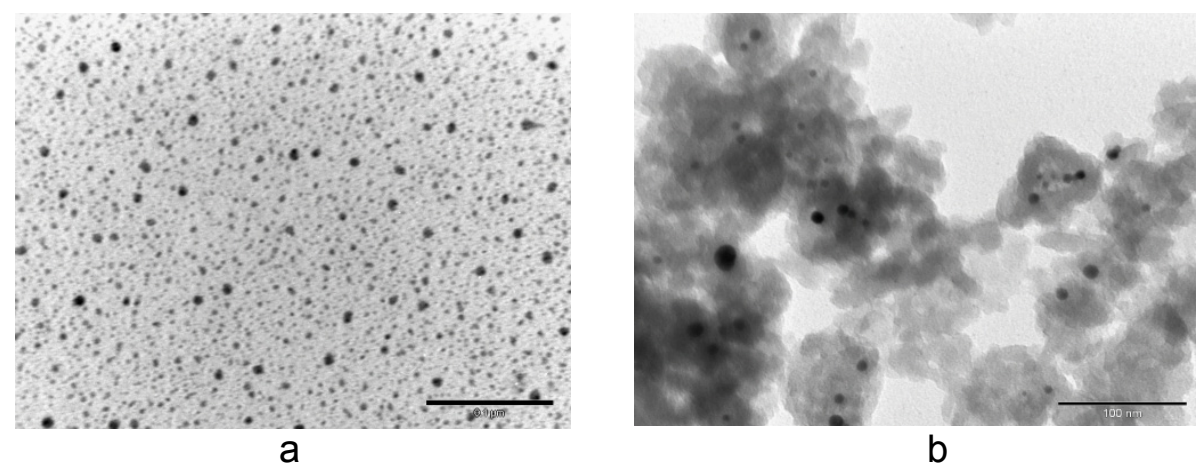

Figure 1. TEM characterization of the Fe@Au nanoparticles and Fe@Au hybrid composites: (a) image of FA1 sample and

(b) TEM imagine of the FA1 covered with PPy-NHS copolymer. 
The TEM images of the Fe@Au nanoparticles were used to determinate the size distributions of the diameters. At least 400 nanoparticles were considered for each sample.

The normalized size distributions of the Fe@Au samples are presented in Fig. 2. One can observe that the mean diameters of the Fe@Au nanoparticles are around 5-7 nm. Due to the superparamagnetic behavior of these systems the size distributions of nanoparticles play an important role for the adjustment of their magnetic properties.

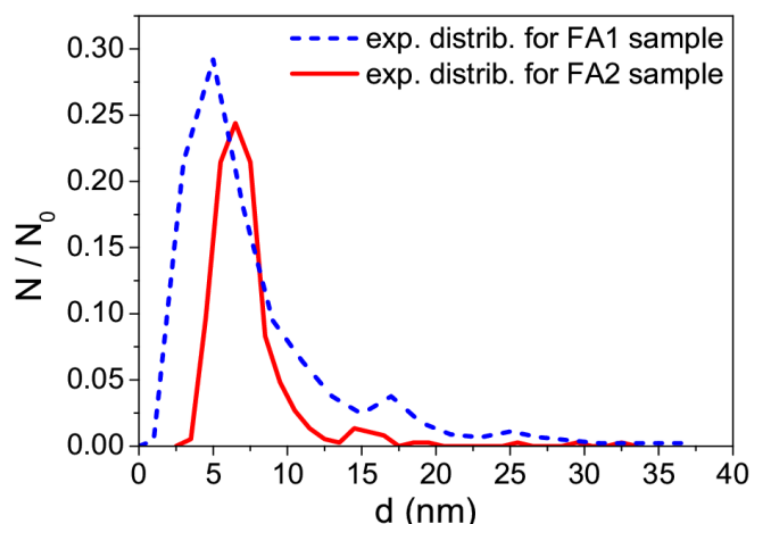

Figure 2. The distributions of the diameters of the nanoparticles for FA1 and FA2 samples.

In Fig. 3 it is presented the UV-Vis spectrum of Fe@Au nanoparticles (FA2 sample for instance). It can be observed the characteristic peak at $553.8 \mathrm{~nm}$ which represents the extinction of the plasmonic mode at the gold surface of nanoparticles. This value appears red shifted as compared to the pure gold nanoparticles $(526 \mathrm{~nm})$ and proves that the "core-shell" structure for Fe@Au nanoparticles is formed [1].

Further, the FTIR technique was used to determine whether or not the PPy-NHS copolymer is formed around the Fe@Au nanoparticles. In Fig. 4 it is presented the FTIR spectra of FA1-PPy-NHS and FA2-PPy-NHS. For comparison, the spectrum of the Py-NHS monomer it is also shown. The spectra of the hybrid composites contain the characteristic absorption bands due to pyrrole ring vibrations located at $914,1190,1465 \mathrm{~cm}^{-1}$ and the band located around $1700 \mathrm{~cm}^{-1}$, ascribed to $\mathrm{C}=\mathrm{O}$ group from the functionalized pyrrole. The presence of the mentioned above bands into the hybrid composites spectra demonstrates the formation of the copolymer between pyrrole and pyrrole functionalized with $\mathrm{N}$-succinimide covering the Fe@Au nanoparticles. A 
frequency shift of the $\mathrm{C}=\mathrm{O}$ band $\left(1737 \mathrm{~cm}^{-1}\right)$ to lower wave numbers $\left(1707 \mathrm{~cm}^{-1}\right)$ is observed in the spectra of the composites samples as compared with that one of the $\mathrm{N}$-succinimide substituted monomer. Possible hydrogen bonding between pyrrole rings and $\mathrm{N}$-succinimide radicals can appeared.

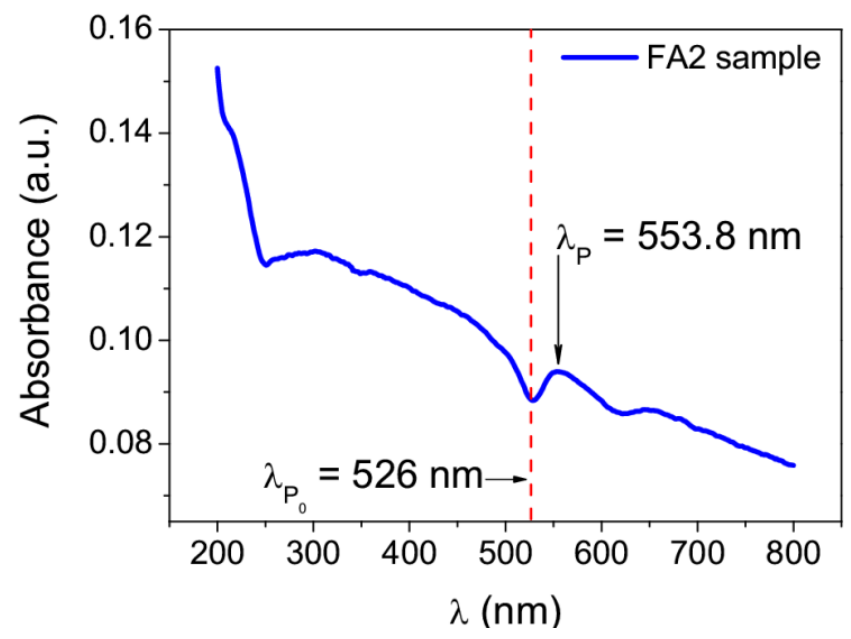

Figure 3. UV-Vis spectrum of FA2 sample.

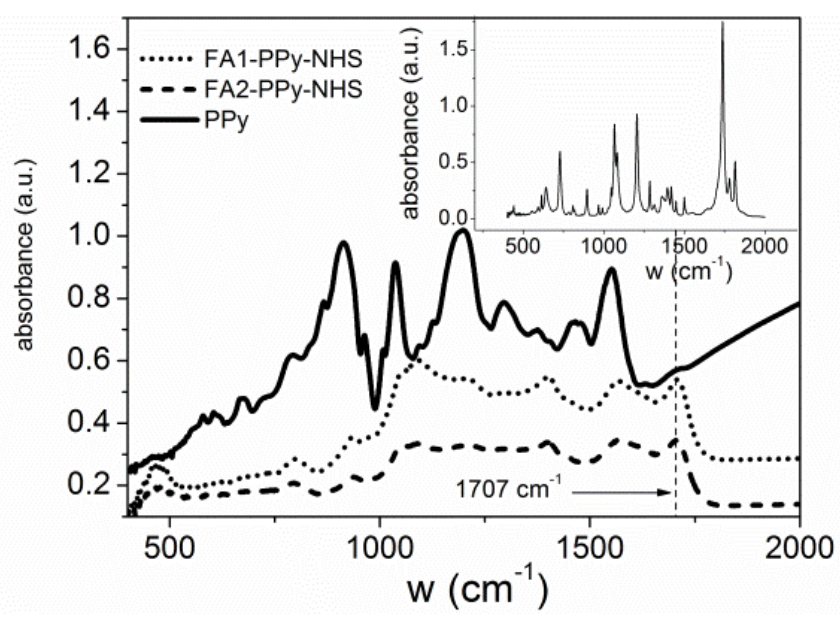

Figure 4. FTIR spectra of hybrid nanocomposites Fe@Au-PPy-NHS compared with the FTIR spectrum of polypyrrole. The inset shows

FTIR absorbtion spectra of Py-NHS monomer. 
On the other hand, the spectra of the two hybrid nanocomposites containing functionalized polypyrroles show significant changes concerning the relative peak intensities and the peak positions as compared to the typical polypyrrole. Therefore, the pyrrole ring vibrations bands located at 914, 1190, $1465 \mathrm{~cm}^{-1}$ in PPy are shifted to higher wave numbers in the PPy-NHS functionalized cases.

Furthermore, the absorption bands ascribed to the collective vibration mode of intra-ring and inter-ring $\mathrm{C}=\mathrm{C} / \mathrm{C}-\mathrm{C}$ are shifted from $1550 \mathrm{~cm}^{-1}$ in $\mathrm{PPy}$ to $1569 \mathrm{~cm}^{-1}$ in magnetic nanoparticles FA1-NHS and $1574 \mathrm{~cm}^{-1}$ for FA2-NHS, respectively. The ring charge distribution modification can be responsible for this effect.

Further, the hybrid nanostructures Fe@Au covered with copolymer functionalized with $\mathrm{N}$-succinimide could be used for the attachment of various bioentities (such as: proteins) that contains $\mathrm{NH}$ - or $\mathrm{SH}$ - groups by a substitution reaction of $\mathrm{N}$-succinimide. Therefore, this property makes this material suitable for in vitro applications like magnetic separation of biomolecules and cells, or in vivo applications as magnetically driven drug delivery.

\section{CONCLUSIONS}

The Fe@Au nanoparticles were obtained by reversed micelles method and these were introduced in copolymer pyrrole and pyrrole functionalized with $\mathrm{N}$-succinimide.

The distribution of the Fe@Au particle sizes was determined from TEM images; the mean diameter is around 5-7 nm.

The characteristic red shift observed for the plasmonic extinction, as indicated in the UV-Vis spectrum of Fe@Au nanoparticles (526 to $553 \mathrm{~nm}$ ), also proves that the "core-shell" structure for Fe@Au nanoparticles is formed.

By using the FTIR spectroscopy it was shown that the $\mathrm{N}$-succinimide functionalized copolymer was attached to the Fe@Au nanoparticles; this conclusion is also sustained by the TEM images of the hybride nanocomposite material. The magnetic behaviour of these samples is under investigation.

\section{EXPERIMENTAL SECTION}

\section{Materials}

The used substances were purchased from different companies: $\mathrm{FeSO}_{4}$ from Chimopar (Romania), $\mathrm{HAuCl}_{4}$ ("Raluca Ripan" Institute for Research in Chemistry, Romania), $\mathrm{NaBH}_{4}(12 \%$ aq. sol. in $14 \mathrm{M} \mathrm{NaOH}$, Aldrich), 
cetyltrimethylammonium bromide (Sigma), pyrrole (97\%, Merck), ammonium persulfate ( $\geq 98 \%$, Sigma-Aldrich), N-hydroxysuccinimide (98\%, Sigma-Aldrich). All the solvents were from Chimopar (Bucharest, Romania). All chemicals were of analytical grade.

\section{Methods}

The iron-gold core-shell nanoparticles (Fe@Au) were prepared by reverse micelle method using cetyltrimethylammonium bromide (CTAB) as surfactant and 1-butanol as co-surfactant. The oil phase was octane.

An amount of $2.4 \mathrm{~mL} 0.8 \mathrm{M} \mathrm{FeSO}_{4}$ (aq.) and $2.4 \mathrm{~mL} \mathrm{NaBH} 41 \mathrm{M}$ were mixed together under magnetic stirring and inert atmosphere, for $1 \mathrm{~h}$. In this reaction was obtained $\mathrm{Fe}^{0}$ (core material). A gold shell was added in aim to protect the iron core. In this goal, the micelles were obtained by adding a solution of $1.5 \mathrm{~mL} \mathrm{NaBH} 41.6 \mathrm{M}$, followed by corresponding quantity of 0.44 $\mathrm{M} \mathrm{HAuCl}_{4}$ (Table 1), in 1-butanol and octane. The mixture obtained was stirred at room temperature under inert atmosphere for $5 \mathrm{~h}$. After synthesis, the remaining surfactant was removed by washing with a $1: 1(\mathrm{v} / \mathrm{v})$ chloroform/ methanol mixture. The powder obtained was dried at $60^{\circ} \mathrm{C}$, in an oven. Further the resulting Fe@Au nanoparticles were covered by copolymer between pyrrole and pyrrole functionalized with $\mathrm{N}$-succinimide.

Table 1. The conditions of Fe@Au nanoparticles synthesis.

\begin{tabular}{|l|c|c|c|}
\hline Sample & \multicolumn{3}{|c|}{ Molar ratios } \\
\cline { 2 - 4 } & $\begin{array}{c}\text { surfactant : } \\
\mathrm{HAuCl}\end{array}$ & $\begin{array}{c}\text { surfactant : } \\
\mathrm{FeSO}_{4}\end{array}$ & $\begin{array}{c}\mathrm{FeSO}_{4}: \\
\mathrm{HAuCl}\end{array}$ \\
\hline $\mathrm{FA}^{\mathrm{a}) 1}$ & 10.4 & 14 & 1.5 \\
\hline $\mathrm{FA} 2$ & 12.5 & 14 & 1.8 \\
\hline
\end{tabular}

a) $\mathrm{FA}=\mathrm{Fe} @ \mathrm{Au}$

The hybrid composites with Fe@Au nanoparticles covered by the pyrrole copolymer were obtained by chemical oxidative polymerization of a mixture of pyrrole (Py) and substituted pyrrole with $\mathrm{N}$-succinimide (Py-NHS) monomers in water. The oxidation agent used in this reaction was ammonium persulfate (APS). Two weight ratios between the monomers (Py, Py-NHS) and Fe@Au nanoparticles were used, namely 1.6 and 0.16 for the synthesis of the sample named FA1-NHS1, and sample FA1-NHS2 respectively. The 
molar ratio between APS and Py-NHS was 0.5 . The reaction time was 10 hours and during this time the solution was kept under sonication. Finally, the co-polymerization reaction was stopped with methanol. The as resulted black precipitate was washed with water. It was separated from the solution by centrifuging and by drying at $100^{\circ} \mathrm{C}$ in an oven.

The samples obtained (Fe@Au-NHS) were analyzed by transmission electron microscopy (TEM) and optical spectroscopic methods (UV-Vis, FTIR).

TEM analysis was performed on $1010 \mathrm{JEOL}$ transmission electron microscope, and UV-Vis analysis on an ABL\&E Jasco V550 spectrophotometer. FTIR measurements were done with a 6100 JASCO spectrometer in the 4000 to $400 \mathrm{~cm}^{-1}$ spectral range with a resolution of $2 \mathrm{~cm}^{-1}$ using the well-known $\mathrm{KBr}$ pellet technique.

\section{ACKNOWLEDGMENTS}

Financial assistance provided through the Ministry of Education and Research of Romania (Research Programs, Projects: ID-76, ID-119) is gratefully acknowledged.

\section{REFERENCES}

1. M. Li, H. Schnablegger, S. Mann, Nature, 1999, 402, 393.

2. J. Chatterjee, Y. Haik, C.J. Chen, Journal of Magnetism and Magnetic Materials, 2002, 246, 382.

3. J.M. Vargas, L. M. Socolovsky, M. Knobel, D. Zanchet, Nanotechnology, 2005, 16, S285.

4. F.M. Mulder, R.C. Thiel, K.H.J. Buschow, Journal of Alloys and Compounds, 1995, 223, 127.

5. E. Carpenter, C.T. Seip, C.J.O'Connor, Journal of Applied Physics, 1999, 85, 5184.

6. L. Néel, Comptes Rendus de l'Académie des Sciences Paris, 1949, 228, 664.

7. N. Sounderya, Y. Zhang, Recent Patents on Biomedical Engineering, 2008, 1, 34.

8. Y.H. Huang, C.H. Yan, Z.M. Wang, C.S. Liao, G.X. Xu, Solid State Communications, 2001, 118, 541.

9. W.F. Jr. Brown, Physical Review, 1963, 130, 1677.

10. J. Lin, W. Zhou, A. Kumbhar, J. Wiemann, J. Fang, E.E. Carpenter, C.J. O'Connor, Journal of Solid State Chemistry, 2001, 159, 26.

11. E.E. Carpenter, C. Sangregorio, C.J. O'Connor, IEEE Transaction on magnetic, $1999,35,3496$. 
12. Y. Bao, K.M. Krishnan, Journal of Magnetism and Magnetic Materials, 2005, 293, 15. 13. W.L. Zhou, E.E. Carpenter, J. Lin, A. Kumbhar, J. Sims, C.J. O'Connorl, European Physical Journal D, 2001, 16, 289.

14. T.T.H. Pham, C. Cao, S.J. Sim, Journal of Magnetism and Magnetic Materials, 2008, 320, 2049.

15. P. Jeffrey, Polymer Reviews, 2007, 47, 231. 